\title{
Decision-Making Optimization Design of Enterprise Standardization Management Planning Based on Mobile Network System
}

\author{
Qiao Wang $\mathbb{D}^{1}{ }^{1}$ Jianjun Wang, ${ }^{2,3}$ and Shenlin $\mathrm{Ye}^{4}$ \\ ${ }^{1}$ School of Humanities and Arts, Xingzhi College of Xi'an University of Finance and Economics, Xi'an, 710038 Shaanxi, China \\ ${ }^{2}$ Administration Office, Safety Supervision Station of Agricultural Machinery in Qindu District, Xianyang, 712099 Shaanxi, China \\ ${ }^{3}$ Auditing Practices Department, China Quality Mark Certification Group Shaanxi Co., Ltd., Xi'an, 710048 Shaanxi, China \\ ${ }^{4}$ Research and Development Department, Pinduoduo Inc., Shanghai 200050, China
}

Correspondence should be addressed to Qiao Wang; wangqiao26708@163.com

Received 13 January 2021; Revised 15 February 2021; Accepted 20 May 2021; Published 1 June 2021

Academic Editor: Wenqing Wu

Copyright (c) 2021 Qiao Wang et al. This is an open access article distributed under the Creative Commons Attribution License, which permits unrestricted use, distribution, and reproduction in any medium, provided the original work is properly cited.

With the continuous deepening of enterprise management and market competition, the pressure of production and operation of enterprises is increasing, and it is urgent to improve the management level of enterprises. This paper mainly studies the decision optimization design of enterprise standardization management planning based on mobile network system. In this paper, the idea of clustering is used to integrate the customers into a customer clustering mode, which will greatly reduce the number of customers studied in the model and simplify the optimization process of the model. Through online and offline processes, these can achieve dynamic and rapid processing of data flow and can well meet the needs of users for data flow analysis. In the construction of enterprise standardization strategic performance evaluation index system, it is necessary to decompose, combine, and converge the index system. According to the construction principles and methods discussed above, the influencing factors of standardized strategic performance evaluation indicators of construction enterprises are preliminarily stratified by using the expert survey method and cluster analysis method, so as to construct the initial evaluation system. For the mobile network system testing, we use the black box test and white box test. The test content mainly includes two modules: area calculation and label calculation. Experimental data show that when the time threshold Ts is set to 1 hour, the prediction accuracy of TBM increases from $46.9 \%$ to $47.7 \%$. The results show that the mobile network system realizes the whole life cycle management of enterprise standards and improves the management level of enterprise standardization work.

\section{Introduction}

Only through the improvement of standardized management level of enterprises, through standardization to guide production, improves product quality and service quality, in order to stand out in such fierce competition, to achieve long-term development of enterprises. However, at present, because many enterprises have not realized the importance of standard informatization, or have not seen the needs of enterprises in this respect, the application examples of enterprise standardized information management system are not many, which can only learn from the experience of other information management systems for system development.
The research in this article enables decision makers to better use scientific decision-making methods to participate in the coordination activities between enterprises in the future, to maximize the profits of the supply chain, and to enhance the overall competitiveness of the supply chain. With the gradual development of the application of the performance analysis system and the full utilization of its functions, it will surely create important value for the enterprise and enhance its core competitiveness. For core enterprises, by helping small- and medium-sized enterprises in the supply chain to solve financing problems, it is conducive to maintaining sustained and stable trade relations and promoting the healthy development of the supply chain. 
The mobile network plays a role in promoting the optimization of the enterprise's standardized management system. Choi proposed a millimeter wave- (MMW-) based mobile hotspot network (MHN) system for high-speed railways, which can support a peak backhaul link throughput of $1 \mathrm{Gbps}$ per train at a speed of $400 \mathrm{~km} / \mathrm{h}$. The MHN system can be implemented in subways and high-speed trains to support passengers and provide access to the Internet through smart devices. The system he proposed can overcome the inherent high path loss of MMW through system design and high antenna gain. He also demonstrated the feasibility of using the test bed deployed in Seoul Metro Line 8 for the MHN system. There are some subtle loopholes in his research [1]. Jao believes that as the demand for newgeneration mobile networks continues to grow, the ITU radiocommunication sector (ITU-R) has proposed key technical performance requirements for the development of IMT-2020 systems and has begun to invite submissions for IMT-2020 candidates radio interface technology. Systemlevel simulation is used as the main tool to evaluate the performance of various application scenarios. Unlike link-level simulation, in link-level simulation, only the link between a base station (BS) and user equipment (UE) is evaluated, while system-level simulation simulates a large number of BSs and UEs in a wide service area. There is no clear direction for his research [2]. Bifulco believes that the service function chain is expected to simplify the process of introducing new services into the mobile network by enabling the dynamic combination of virtualized network functions. However, the current implementation relies on new tunneling protocols and changes in network infrastructure, which makes deployment in traditional networks difficult. He proposed a system for service function chains that can be easily deployed in mobile networks without any protocol or network modification. In addition, he proposed the design of a hybrid hardware-software software-defined network switch to implement a scalable network traffic classifier, which CATENAE uses to allocate network flows to corresponding functional chains. His research lacks necessary experimental data [3].

This paper mainly discusses the optimal design of enterprise standardized management planning decision-making under mobile network system and adopts the visual way to support the collaborative modeling process of value model and evaluation system. The evaluation results are displayed in a variety of graphical forms, and the local or global view can be viewed by zooming the view. The system makes full use of factory mode and multithread control technology to make the overall structure of the module clear and concise, and ensure the subsequent maintainability and scalability of the module.

\section{Mobile Network System and Enterprise Standardized Management}

2.1. Mobile Network System. The Manhattan distance function follows a grid-like path to calculate the distance from one data point to another. The Manhattan distance between two data points is the sum of the grid paths [4]. The formula for the distance between point $X=\left(X_{1}, X_{2}, \cdots, X_{n}\right)$ and point $Y=\left(Y_{1}, Y_{2}, \cdots, Y_{n}\right)$ is as follows:

$$
d=\sum_{i=1}^{n}\left|X_{i}-Y_{i}\right|
$$

The Euclidean distance function measures the straight line distance between two points. The formula for the distance between point $X=\left(X_{1}, X_{2}, \cdots, X_{n}\right)$ and point $Y=$ $\left(Y_{1}, Y_{2}, \cdots, Y_{n}\right)$ is as follows:

$$
d=\sqrt{\sum_{i=1}^{n}\left(X_{j}-Y_{j}\right)^{2}} .
$$

The overall architecture design of the mobile network system is shown in Figure 1.

The objective function is defined as follows:

$$
J=\sum_{k=1}^{K} \sum_{x_{i} \in D_{k}}\left\|x_{i}-c_{k}\right\|^{2}
$$

If the sample has the smallest distance from the center $c_{k}$ of the $k$-th category, the sample belongs to the cluster $D_{k}$, which can be described as follows:

$$
\begin{gathered}
D_{k}=\left\{x_{i} \in X \mid k=\underset{j \in\{1,2, \cdots, k\}}{\arg \min }\left\|x_{i}-c_{j}\right\|^{2}\right\}, \\
c_{k}=\frac{\sum_{x_{i} \in D_{k}} x_{i}}{\left|D_{k}\right|} .
\end{gathered}
$$

The weight calculation formula is defined as follows:

$$
W_{A_{i}}=\frac{N_{A_{i}}}{\sum_{j=1}^{n} N_{A_{j}}} .
$$

The calculation formula of the clustering coefficient of node $v 2$ is as follows:

$$
c c=\frac{n}{\left(\begin{array}{l}
k \\
2
\end{array}\right)}=\frac{2 n}{k(k-1)} .
$$

In the formula, $k$ represents the number of all adjacent nodes of node $v 2$ [5]. The calculation method of the cosine similarity between node $i$ and node $j$ is as follows:

$$
\cos =\frac{a_{i} \cdot a_{j}}{\left\|a_{i}\right\| \cdot\left\|a_{j}\right\|} .
$$

Among them, $\|\cdot\|$ means two norms. The similarity of network nodes is widely used in the clustering of networks and networked data. For example, the similarity between social network nodes can be used to recommend friends [6]. 


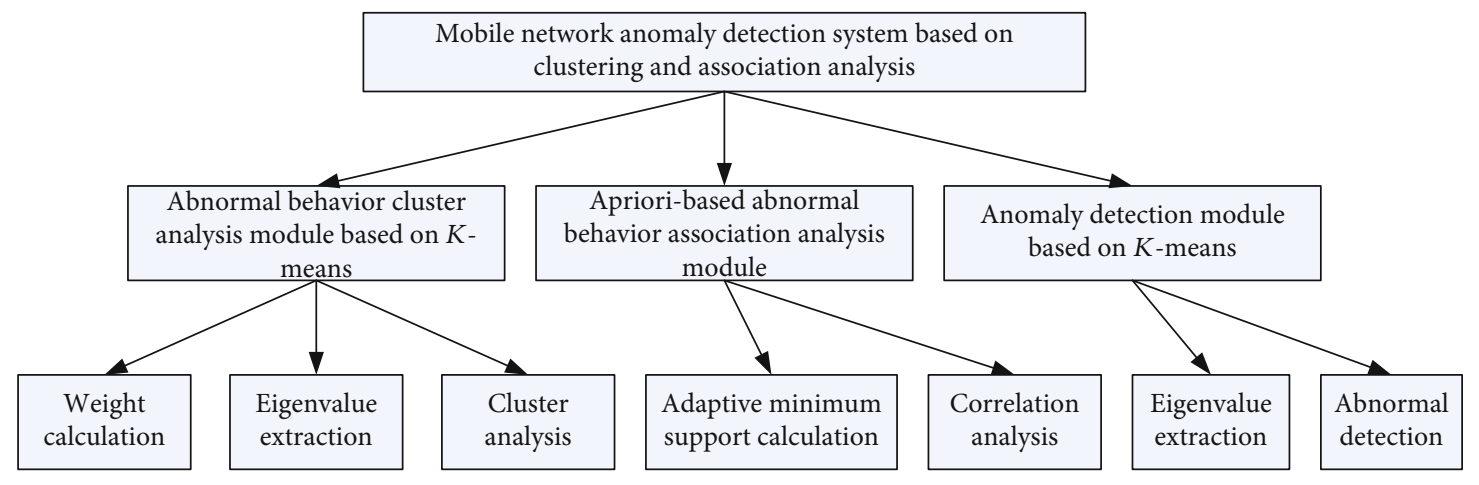

FIGURE 1: The overall architecture design of the mobile network system.

The probability of a network with $n$ nodes and $m$ edges obeys the binomial distribution shown in the following formula:

$$
P(m)=\left(\begin{array}{c}
\frac{n(n-1)}{2} \\
m
\end{array}\right) p^{m}(1-p)^{n(n-1) / 2-m} .
$$

The expected value of $m$ is as follows:

$$
\langle m\rangle=\sum_{m=0}^{n(n-1) / 2} m P(m)=p \cdot \frac{n(n-1)}{2} .
$$

The average degree of random network $\langle k\rangle$ is calculated as follows:

$$
\langle k\rangle=\frac{2\langle m\rangle}{n}=\frac{2 p \cdot n(n-1) / 2}{n}=p \cdot(n-1) .
$$

The calculation method of the degree distribution of a random network is as follows:

$$
P(k)=\left(\begin{array}{l}
n \\
k
\end{array}\right) p^{k}(1-p)^{n-k} \approx e^{\langle k\rangle} \cdot \frac{\langle k\rangle^{k}}{k !} .
$$

The platform monitors Internet traffic data through the self-developed traffic monitoring system TMS, which is deployed between the access network and the core network. Whenever a user uses the device to go online, TMS can collect all the upstream and downstream data packets and generate streams through collection recording. These stream records are distributed to the data processing module through the data distribution system [7]. The calculation results of batch processing and real-time processing can be stored in a relational database. The platform provides a friendly interface module to display these results. In addition, the cluster monitoring module is used to monitor the abnormality of the platform. It can collect the performance indicators of the hardware and software in the cluster. When errors and alarms occur, the cluster manager will notify the cluster manager through emails and text messages for processing [8].
2.2. Enterprise Standardization Management. Enterprise standardization information management system is based on the relevant information of enterprise standardization. After the full analysis and investigation of enterprise standardization management, the system of standardized information network is established based on the development goal of the enterprise, and the actions of various departments of the enterprise are connected in series, so as to enjoy the effective influence brought by standardization and maximize the benefits of the enterprise. A number of standards are organically combined and complemented to form a relatively perfect logistics standard system [9].

The logistics standard unit formed in the process of logistics enterprise management standardization can provide factual support and experience reference for logistics industry standardization, and logistics industry standardization, as a verified standard reference, can be applied to logistics enterprise standard unit, thus indirectly promoting logistics enterprise standardization management. But at the same time, the concept of logistics standardization can also be applied to the standardization management of enterprises based on nonlogistics business standards, playing a reference significance $[10,11]$.

In addition, with the development of computer monitoring technology and the improvement of automation degree of enterprise management information system, hydropower enterprises must timely adjust the original equipment management organization form and build a flat, crossdepartmental matrix equipment management mechanism as soon as possible. In fact, when people really get a sense of accomplishment and satisfaction from their heart, that kind of enthusiasm will promote better completion of tasks. Therefore, hydropower enterprises should improve the living and working environment of employees, quantify objectives and tasks, and set up effective commendation methods, so that enterprises can make full use of their talents and employees can obtain a certain sense of achievement through work $[12,13]$.

The stronger the competitiveness of an enterprise, the higher its status, the higher its market visibility, and the higher the probability of establishing a corresponding relationship. The strong competitiveness and high status of an enterprise reflect the company's ability to solve problems and the current good operating conditions of the enterprise. 
This also means that the knowledge and experience that the enterprise has stored in the market operation is quite rich. With the deepening of the degree of interaction between the core enterprise and the related organizations in the relationship network, it will help the core enterprise to lock the organization in the relationship network [14].

When the two parties to establish a relationship have a deeper understanding of each other, the two parties will carry out more in-depth cooperation around technology standardization, and there will be a certain degree of path dependence between each other. Therefore, relevant organizations such as intermediary, business, scientific research, and administration will be locked in the relationship network established by the core enterprises. An important prerequisite for the stable operation and development of relational network is that all the member organizations in the network can get the return corresponding to their efforts. When a member gains noncorresponding benefits, it will harm the interests of other members, thus causing other members to have reservations in the cooperation [15].

2.3. Decision Optimization System. The decomposition of engineering product design objects is actually the decomposition of complex product multidisciplinary design decision problems into multiple relatively simple disciplines or subsystems, and each discipline or subsystem can be analyzed and optimized in a relatively independent environment at the same time. This paper uses the fuzzy clustering method to divide the strategy set of each game party [16].

Single objective optimization is performed on $n$ objective functions separately, so as to obtain the optimization vector of each objective function when the optimal value is obtained:

$$
X_{j}^{*}=\left\{x_{1 j}^{*}, x_{2 j}^{*}, \cdots, x_{m j}^{*}\right\}, \quad j=1,2, \cdots, n .
$$

In the formula, $X_{j}^{*}$ is the set of design variables corresponding to the $j$-th design goal when the optimal value is obtained.

DPI is defined as the expected value of the design performance preference function within the design solution range, namely,

$$
\mathrm{DPI}=E[P(y)]=\int_{\bar{y}-\Delta y}^{\bar{y}+\Delta y} P(y) f(y) d y .
$$

The decision support system uses local branch and delimitation, tabu search, and neural network algorithms according to different underground roadway environment and inspection area status and other information to calculate the path planning of underground security personnel in different scenarios and different scales, and calculates the parking of each person and vehicle. Points need to arrange the number of security personnel, and the safety inspection system generates a safety inspection route for each security personnel, and can observe the entire downhole situation in real time through the personnel positioning module, and can conduct two-way early warning up and down $[17,18]$.
According to this platform, it can quickly and accurately calculate the inspection path planning of mine security personnel, and carry out positioning and early warning, so as to improve the mine safety and personnel work efficiency and reduce enterprise costs. The fitness function requires the planning scheme to make the objective optimal under the constraint conditions. In the fitness function, it is required to reflect both the expected factors of the solution and the undesirable factors of the solution, and reward the former and punish the latter [19]. Generally, the fitness function adopts the maximum principle; that is, the greater the fitness value, the better the variety. After the main grid positions are initially determined, the power flow of the network is calculated with the minimum line loss as the optimization objective, meeting the constraints of power supply radius and voltage loss, and the line loss of different grid structures is compared and analyzed, and finally, a new grid structure is obtained [20,21].

\section{Mobile Network System Simulation Experiment}

3.1. Test Environment. Considering the common hardware environment in current practical application, we use IBM System x3650 M2 server as web server and database server, and select two smart phones for mobile terminal equipment. We use Windows Server 2008 as the operating system of the server, Tomcat as the web server, and MySQL database as the most common database [22]. The system simulation parameter settings are shown in Table 1.

3.2. Data Preprocessing. This paper adopts the idea of clustering, integrates customers into a customer clustering model, and gathers multiple small customers into one big customer, which will greatly reduce the number of customers studied in the model, thereby simplifying the optimization solution process of the model. Based on the principle of minimizing logistics costs, the distribution of production locations to customers often follows the principle of nearby distribution, and because the capacity of each production location is limited, it can only meet the needs of some nearby customers, so in order to reduce the scale of the problem, it can be based on the production location. Geographic location and historical data of distribution, the production area, and customers are divided into regions, the production area in each area mainly supplies the needs of customers in the area, and the remaining production is used for external supply. Through this division of the main supply area, the large-scale resource allocation problem is divided into several smaller-scale resource allocation problems $[20,23]$.

3.3. Data Stream Clustering. The data stream clustering of the mobile network system is divided into two parts: an online process that records the current data stream clustering characteristics and an offline process for users to perform offline queries. The online process quickly receives the input data stream, and saves the clustering results it produces as the intermediate results of mining for users to query offline. Through online and offline processes, dynamic and fast data 
TABLE 1: System simulation parameter settings.

\begin{tabular}{lc}
\hline Simulation parameters & Simulation value \\
\hline Number of SDP sites M & 3 \\
Number of functional modules per SDP site N & 10 \\
IoT business category K & 6 \\
IoT aggregation length L & 10 \\
IoT aggregation delay threshold T & $1 s$ \\
\hline
\end{tabular}

flow processing is realized, and users' needs for data flow analysis can be well met [24].

(1) Initialize the data stream. At the beginning, several data are read in at one time, and divided to form an initial grid, and calculate the feature vector of each grid unit

(2) Add new data objects. With the inflow of new data objects, each data point will be located in the corresponding spatial grid according to its own information, and the feature vector of the grid unit will be updated in real time. Due to the large amount of data flow and unpredictability, if you store all the grids, a lot of storage space is required. In order to save storage space, in this algorithm, we only store grid cells with data points [25]

(3) Mobile nonintensive units. Due to the complementary and overlapping division of the grid, some of the influence of the data points on the surrounding space is lost, and at the same time, the data points that originally belong to a certain category may be unevenly distributed into different grids. This effect has on the grid unit. The edge data is particularly obvious. After the grid unit is moved, a new grid unit is generated, and the grid unit vector and its density are calculated. At this time, it is not necessary to scan the entire data set, as long as the data unit adjacent to the grid unit is viewed [26]

(4) Update the feature vector of the grid unit. Under the condition of continuous data flow, after moving the entire data space, the data space is composed of the original dense unit and the newly added unit

(5) Identify clusters. After moving the nondense grid cells, the data space is composed of the original dense grid cells and newly added grid cells. According to the depth-first traversal algorithm to find the related dense units, find all the dense units associated with the unit and merge these units to generate a cluster [27]

3.4. Construction of Evaluation Indicators. In the construction of enterprise standardization strategic performance evaluation index system, it is necessary to decompose, combine, and converge the index system. In the performance of standardization strategy of construction enterprises, process and result are equally emphasized; that is, in the design of index system, on the one hand, the final result of strategy implementation is emphasized; on the other hand, the process of strategic evaluation, selection, and implementation is also emphasized. According to the construction principles and methods discussed above, the influencing factors of standardized strategic performance evaluation indicators of construction enterprises are preliminarily stratified by using the expert survey method and cluster analysis method, so as to construct the initial evaluation system [28].

(1) Select 9 experts to score the 5 elements in the selected evaluation system and quantitatively score them according to their contribution to the evaluation target. The scores are, respectively, 5, 4, 3, 2, 1

(2) Analyze several elements with a small total score of the expert's score, and when it is determined that its contribution to the evaluation target is small or that it can be merged into other indicators, remove the indicator and perform cluster analysis on the remaining indicators

(3) Use SPSS software to perform cluster analysis, connect factors with similar properties in a step-by-step connection method, and cluster all influencing factors

(4) After the cluster analysis is completed and experts' opinions are consulted again, the preliminary index system of performance evaluation for standardization strategy of construction enterprises can be obtained. Finally, six parts are selected in the initial system indicators of construction industry standardization strategy evaluation, including standardization strategy planning and implementation, customer focus, internal management improvement, learning and growth, project management improvement, and economic benefit promotion [29]

\section{System Simulation Analysis}

4.1. Application Analysis of Enterprise Standard Management. The training set and test set data are sequentially input into the support vector regression machine, decision tree, and random forest for training. The training effect of the proxy model obtained after tuning the parameters is shown in Table 2 and Figure 2. It can be seen that the coefficient of $R^{2}$ when GA is used as an independent variable is 0.98 , which is significantly positive at the $1 \%$ level. It proves that assuming $\mathrm{H} 1$ remains unchanged in other influencing factors, the improvement of business innovation ability in the new economic era indicates that the more effective the enterprise is to help the new economic era through innovative internal control. In addition, the effectiveness of external capital market supervision factors is significantly related to the audit effectiveness, indicating that the quality of the audit system of internal control audit reports has an effect on the effectiveness of corporate internal control, and confirms that further strengthening of internal control audits can promote the role of enterprises in helping the new economic era. 
TABLE 2: Model training effect.

\begin{tabular}{|c|c|c|c|c|}
\hline \multirow{2}{*}{ Model used } & \multicolumn{2}{|c|}{$R^{2}$} & \multicolumn{2}{|c|}{ RMSE } \\
\hline & Training set & Test set & Training set & Test set \\
\hline Support vector regression machine & 0.9774 & 0.971 & 0.9531 & 1.1184 \\
\hline Decision tree & 0.9813 & 0.9673 & 0.867 & 1.187 \\
\hline Random forest & 0.9873 & 0.9732 & 0.7135 & 1.0748 \\
\hline
\end{tabular}

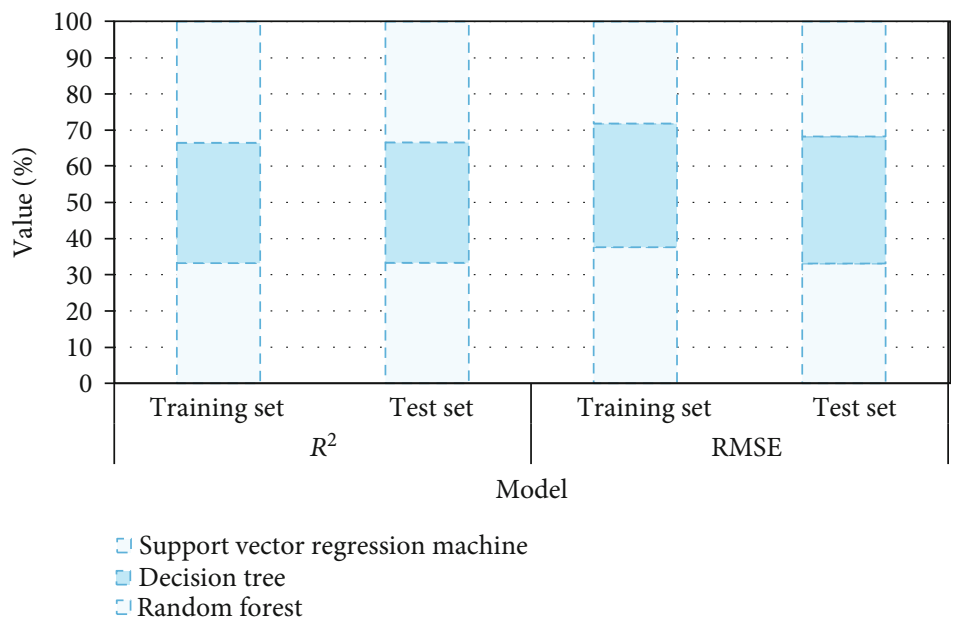

FIGURE 2: Model training effect.

Table 3: Movement characteristics at different times.

\begin{tabular}{lccc}
\hline & $\begin{array}{c}\text { Working } \\
\text { hours }\end{array}$ & $\begin{array}{c}\text { Nonworking } \\
\text { hours }\end{array}$ & Weekend \\
\hline $\begin{array}{l}\text { Location entropy } \\
\begin{array}{l}\text { Radius of } \\
\text { gyration }\end{array}\end{array}$ & 1.71 & 1.81 & 1.97 \\
\hline
\end{tabular}

The generation of decision support systems is based on the theoretical development of management information systems and other related disciplines. Although traditional MIS can organize and manage information on the surface, it cannot dig out the internal laws of information to serve decisionmaking. The help of managers is limited, and the expected socioeconomic effects have not been achieved. The movement characteristics at different times are shown in Table 3. It can be seen from the table that people are more inclined to move during nonworking hours, especially on weekends. Both the entropy and the radius of gyration increase significantly, indicating that people are more mobile at this time. This also illustrates the necessity of proposing time-based mobility algorithms.

We use Markov and TBM to experiment on the prediction accuracy, respectively, and the Ts parameter of TBM is set to 1 hour and 2 hours, respectively. At the same time, the algorithm was tested separately for mobile users and all users. The final experimental results are shown in Table 4 and Figure 3. It is known from the table that TBM can achieve a better prediction effect for mobile users than basic Markov. When the time threshold Ts is set to 1 hour, the prediction accuracy of TBM rises from $46.9 \%$ to $47.7 \%$; when the
TABLE 4: Experimental results.

\begin{tabular}{lcc}
\hline Forecast accuracy & All users & Mobile users \\
\hline Markov & $34.3 \%$ & $46.9 \%$ \\
TBM $(\mathrm{Ts}=1 \mathrm{~h})$ & $36.2 \%$ & $47.7 \%$ \\
$\mathrm{TBM}(\mathrm{Ts}=2 \mathrm{~h})$ & $37.2 \%$ & $48.6 \%$ \\
\hline
\end{tabular}

time threshold Ts is set to 2 hours, higher prediction accuracy can be obtained. In a sense, it is "using the skills of others to do a good job." It is a collaborative activity that gathers people with different personalities, specialties, and preferences under the same organizational goal. The uniqueness of corporate goals determines that corporate standardization managers cannot seek common ground while reserving differences can only give up or change personal views, compromise, and compromise with each other for the common goals of the enterprise. On the other hand, in scientific research, scholars, as knowledge disseminators and research direction masters, generally adopt a condescending, authoritative, and paternalistic management mode for their disciples and students. Enterprise employees have professional skills and have been on the front line of research and development for a long time. They are the creators and specific implementers of innovative activities. Authoritative and too rigid management methods are not conducive to activating employees' thinking and maintaining creativity. Therefore, companies often use incentives: management mode.

4.2. Performance Analysis of Decision Optimization System. Normalized throughput is an important index to measure 


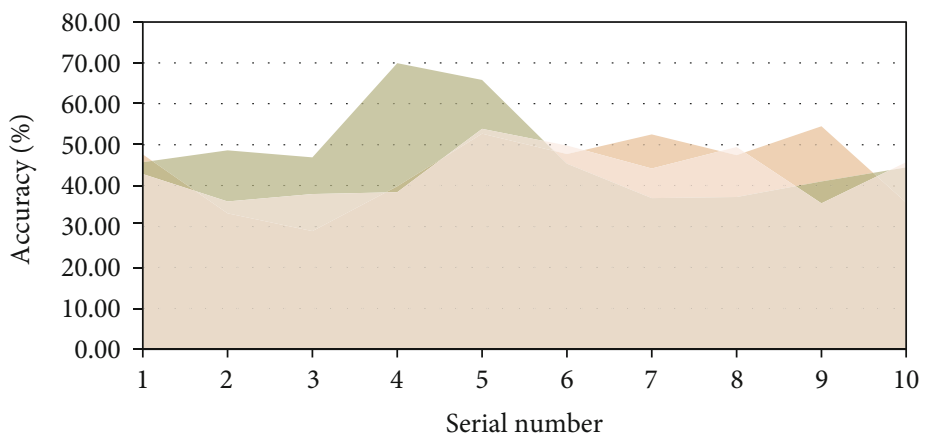

Markov

TBM $(\mathrm{Ts}=1 \mathrm{~h})$

TBM $(\mathrm{Ts}=2 \mathrm{~h})$

FIGURE 3: Experimental results.

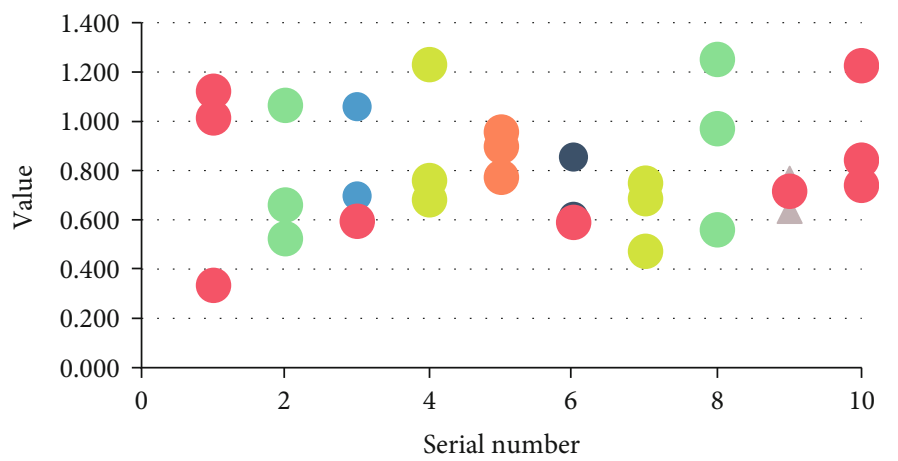

RA-RACH

AC-RACH

FC-RACH

FIgURE 4: Normalized throughput rate under different access methods.

random access, which directly shows the quality of random access process. The high normalized throughput indicates that the system has strong access ability and can drive more devices under the limited time-frequency resources. When the number of RA-RACH users increases to a certain number, serious collision conflicts will occur, resulting in poor access ability. However, AC-RACH and FC-RACH will not have this problem. The normalized throughput under different access modes is shown in Figure 4. It can be seen from the figure that the normalized throughput of the random access mode with AC-RACH and FC-RACH is higher than that of RA-RACH. Especially when the arrival rate of user request is higher and higher, the throughput of RA-RACH access will decrease with collision until it approaches zero. AC-RACH and FC-RACH keep the normalized throughput near the maximum by restraining the real-time demand of some users, so as to realize the system optimization. Compared with AC-RACH, FC-RACH has more feedback to adjust the input and compensate the estimated value of access load to a position closer to the real load.

The comparison of throughput rate between genetic algorithm and assisted call admission algorithm is shown in Figure 5. It can be seen from the figure that the throughput rate of the system increases first as the number of users increases, and then stabilizes. When the number of users is less than 1,000, there is little difference in throughput between GA and MACA. This is because when the number of users is small, the base station has enough resources to serve all users. Although the throughput rate is equal, the resource utilization rate of the base station is different. When the number of users increases again, MACA's system throughput rate will soon reach the upper limit and GA can continue to increase until the number of users is about 2,000 . The final stable system throughput rate of GA is higher than that of MACA. Therefore, laboratory scientific research and enterprise innovation are two completely different fields, and there are many differences in communication management mode, thinking mode, innovation goal, implementation conditions, and so on. These differences make scholars have conflicts of roles in the process of transforming into executives. As a result, scholars with rich scientific research achievements and outstanding scientific research capabilities may not be able to successfully transform into outstanding corporate standardization managers.

The change of recognition accuracy with distance is shown in Figure 6. As can be seen from the figure, when the distance between devices increases, the accuracy of device selection gradually decreases. When the distance between devices is fixed and the angle increases, the accuracy of device selection also increases. Moreover, in the Spartacus system, 


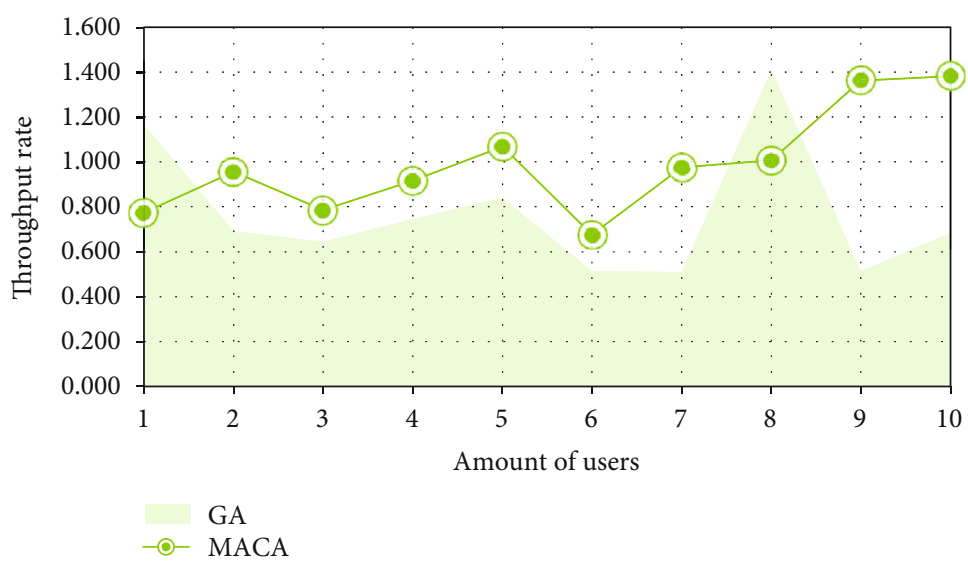

FIGURE 5: Comparison of throughput rate between genetic algorithm and assisted call admission algorithm.

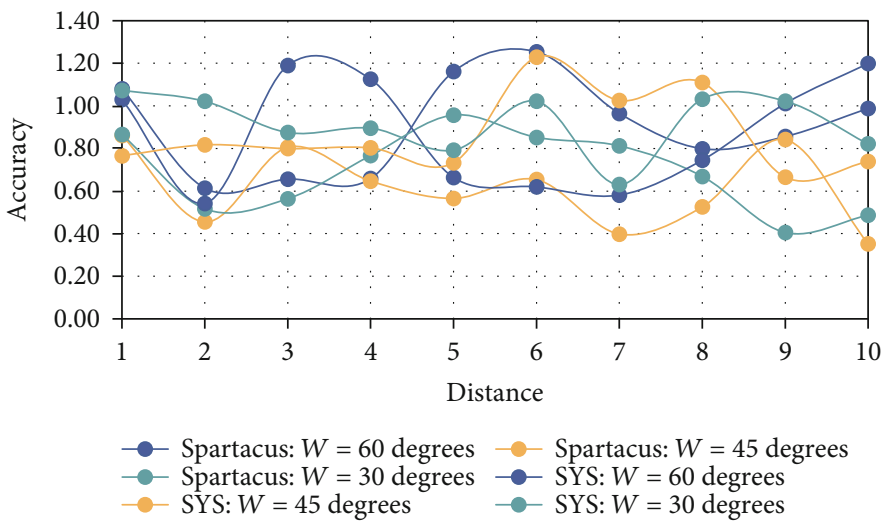

FIGURE 6: Variation of recognition accuracy with distance.

when the angle was 45 degrees and the distance between the source device and the receiving device was less than 4 meters, the accuracy rate could reach $95 \%$. In this system, the same angle and distance, accuracy can be up to 100 percent. When the angle was 30 degrees and the distance was 3 meters, the device selection accuracy of Spartacus could reach $90 \%$, while in the same environment of this system, the accuracy could reach $95 \%$. When the distance between the source device and the receiving device is less than 3 meters, the accuracy of device identification is relatively stable. This is consistent with the phenomenon of sound energy depletion. Because SYS uses energy contrast to detect audio, the energy difference between audio and other bands decreases with distance. Moreover, due to the improved performance generated by arm extension, SYS was able to achieve 95\% accuracy within 3 meters, 90\% accuracy within 4 meters, and 85\% accuracy within 5 meters for all device tests. For enterprise standardization management, there are obvious differences between this management and academic activities in many aspects such as communication methods, thinking modes, innovation goals, and restrictive conditions. This has led to a certain degree of a role conflict between scholars in the process of transforming into corporate executives. The innovation effect of a decisive position such as chairman or CEO is lower than that of other senior management positions, indicating that
TABLE 5: The recognition accuracy of the system in different environments.

\begin{tabular}{lcccccc}
\hline & \multicolumn{5}{c}{ Distance (m) } \\
& 0.5 & 1 & 1.5 & 2 & 2.5 & 3 \\
\hline Hall & $94.5 \%$ & $85.1 \%$ & $82.4 \%$ & $86.7 \%$ & $90.1 \%$ & $92.3 \%$ \\
Laboratory & $97.1 \%$ & $89.5 \%$ & $87.4 \%$ & $88.2 \%$ & $92.1 \%$ & $93.2 \%$ \\
Corridor & $94.8 \%$ & $92.4 \%$ & $97.4 \%$ & $85.2 \%$ & $83.8 \%$ & $86.4 \%$ \\
\hline
\end{tabular}

the innovation promotion effect of academic senior management is closely related to the position of the company.

The recognition accuracy of the system in different environments is shown in Table 5. For a distance range of 0.5 meters, $100 \%$ equipment selection accuracy can be achieved in all three scenarios. When the distance increases, the performance of the hall is basically stable, and the accuracy rate is close to $100 \%$, but the performance of the laboratory and the corridor decreases slightly with the increase of the distance, but the laboratory environment can still guarantee an accuracy of more than $90 \%$, and the corridor environment can still guarantee an accuracy of over $85 \%$. Comparing these two scenes with the lobby, this is mainly due to the powerful multichannel effect. Before training activities, the actual needs of employees should be understood through 
TABLE 6: Descriptive statistics of core corporate capabilities.

\begin{tabular}{lllllllllllll}
\hline & H11 & H12 & H13 & H21 & H22 & H23 & H31 & H32 & H33 & H41 & H42 \\
\hline Mean & 3.28 & 3.97 & 4.36 & 3.69 & 3.77 & 3.69 & 3.61 & 3.89 & 4.23 & 3.45 & 3.77 \\
Median & 3.00 & 4.00 & 5.00 & 4.00 & 4.00 & 4.00 & 4.00 & 4.00 & 4.00 & 3.00 & 4.00 \\
Standard deviation & 0.90 & 0.74 & 0.79 & 0.91 & 0.84 & 1.00 & 0.86 & 0.83 & 0.93 & 0.84 & 0.78 \\
\hline
\end{tabular}

questionnaires, interviews, etc., and the needs of employees should be combined with the needs of the company, so that training can be as close as possible to standardized management and job requirements. Establish scientific and standardized employee training procedures, set up targeted training courses, and help employees improve their work skills and quality in the most efficient manner through the supervision of the training process and the assessment and feedback of the training results.

4.3. Role of Mobile Networks in Corporate Management. The descriptive statistics of core enterprise capabilities are shown in Table 6. In terms of scale, it is considered that 5 billion is an enterprise with certain regulations, with the highest average, reaching 4.36; the smallest degree of dispersion is that one billion is an enterprise with a certain scale, reaching 0.743 ; in terms of development capacity, the average number of high growth enterprises is higher, reaching 3.77 , with a large degree of dispersion, reaching 0.849 ; in terms of leading ability, the average number of high growth enterprises is higher, reaching 3.77 , with a large degree of dispersion, reaching 0.849 . It is considered that the leading power of enterprises with 500 suppliers is strong, with the highest average value of 4.23 . The smallest degree of dispersion is that the core enterprises with more than 200 suppliers have a strong leading ability in the supply chain, reaching 0.843 .

The comparison between the predicted value and the real value of the test set is shown in Figure 7. The critical values of robustness of regular network and scale-free network are both 0 , which means that the robustness of these two types of networks is almost the same. However, the essence of scale-free network is a sparse network; that is, the number of edges of the network is not too many, and the number of edges of regular network is too much; even some regular networks, the network is not sparse enough. When $k=2$, the clustering criterion function value of the improved algorithm is significantly higher than that of the MinMax $k$-means algorithm, but from $k=3$, the clustering criterion function value of the improved algorithm decreases obviously. With the increase of clustering number $k$, the clustering criterion function values of MinMax $k$-means algorithm and improved algorithm tend to be parallel to each other and always maintain a certain gap. Explain that the heterogeneity of enterprises (enterprise establishment time, enterprise profitability, business complexity, asset scale, and actual controller) has a significant impact on the empirical results, and different types of enterprises show the existence of effectiveness of the new era of internal control application economy significant differences. Finally, the three control variables PR, PETIC, and IDR related to the respondent failed to pass the test, indicating that the professional relevance, internal

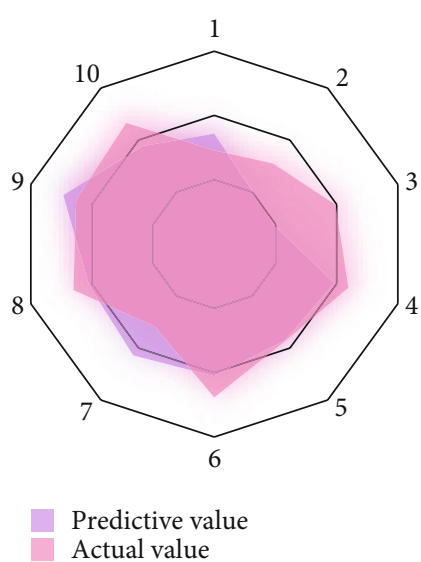

FIgURE 7: Comparison of the predicted value and the true value of the test set.

control experience, and regional differences of different respondents contribute to the business innovation ability and internal control innovation. The relationship between the effectiveness of the new economic era has no impact.

The traffic consumption comparison between MP2PVPN and OpenVPN is shown in Figure 8. The average daily traffic consumed by MP2P-VPN and OpenVPN is $407 \mathrm{kB}$ and $1508 \mathrm{kB}$, respectively. It can be seen that the traffic required to maintain the MP2P-VPN network is only about a quarter of that of OpenVPN, saving a lot of network traffic. The core task of the enterprise standardization strategy that is leading in terms of technical standards is to make its own standards the dominant or de facto standard in the industry. For this reason, it is necessary to find a strategy to make the network effect beneficial to itself and to establish its own installation foundation as soon as possible. Clever use of positive feedback loops attracts consumers to bear the switching costs and ultimately lock the market on its own technology. In the new economic era, the stronger the company's ability to stimulate new power, the more effective it is to help the new economic era through innovative internal control.

\section{Conclusions}

The mobile network automatic drive test system is implemented by $\mathrm{C} / \mathrm{S}$ structure; the interface layer is based on the user-defined control implemented by the third party control and is completely separated from the data based on the event message mechanism; the business logic layer integrates the protocol analysis, signaling process judgment, namely, the use of event finite state machine, and the implementation of network optimization engineering algorithm. The optimized hierarchical structure, the most reasonable modular 


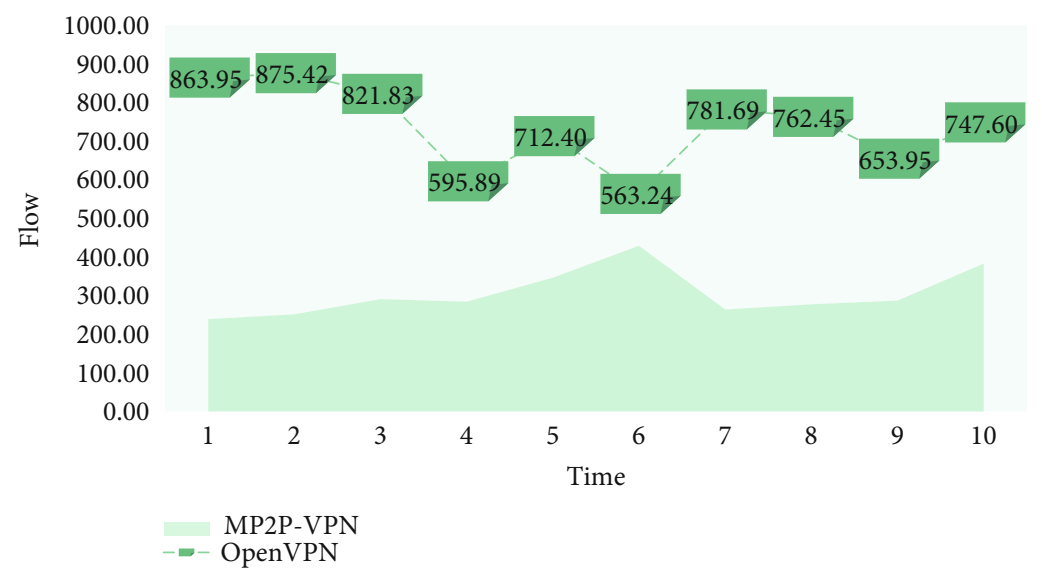

Figure 8: Traffic consumption comparison between MP2P-VPN and OpenVPN.

logic design, and the most rigorous code implementation make the mobile network automatic drive test system have powerful and perfect functions, which can easily and accurately realize the test scheme, analyze the network status, and generate test result reports. It provides comprehensive and accurate data support for network optimization and effectively improves the convenience of system use. The deployment of the whole network optimization system has gone through the process of demand collection, system development, and test acceptance, and most of the test results basically achieve the expected objectives, and the system runs normally.

This paper analyzes the existing research on the coordination and optimization of enterprise production and distribution and the application of decision support system in logistics distribution and production. According to the characteristics of multiorigin, multicustomer, and multiproduct logistics resource allocation problem, combined with the characteristics of decision support system, the framework of decision support system for logistics resource allocation is designed. The coordination and optimization model is established for the logistics resource allocation problem of multiorigin, multicustomer, and multiproduct types, and the DSS system is designed and developed. This paper establishes the general idea, main contents, and development principles of enterprise standardization management system. Through the establishment of the principle of standardization management mode, it specifically expounds the methods for functional departments, grass-roots units, and grass-roots teams to carry out the construction of standard system. The practical tools for functional departments to carry out the construction of standard system are given, which provides guidance and basis for enterprises to carry out standardization management.

The innovation effect of academic executives when they occupy decisive positions in enterprises is not significant when they hold other nondeterministic positions. The increase in the proportion of academic executives does not simultaneously bring about an increase in innovation effects. The above findings help to understand the role of academic executives in corporate innovation activities more rationally and objectively; that is, scientific and technological person- nel's "breaking fence" entrepreneurship and participatory entrepreneurship may be better than dominant entrepreneurship. Therefore, it is recommended that companies do not seek intellectual support from scholars as much as possible. Instead, they should appropriately control the proportion of academic executives, so that they can not only give full play to the professional and resource advantages brought by academic experience but also avoid the "shortcomings" of scholars. The adverse impact is on innovation and entrepreneurship. In addition, enterprises should choose appropriate methods and methods, adopt various forms of "flexibility" to attract talents, make the best use of their talents, and realize the real purpose of hiring academic executives.

\section{Data Availability}

No data were used to support this study.

\section{Conflicts of Interest}

The authors declare that they have no conflicts of interest.

\section{References}

[1] S. Choi, H. Chung, J. Kim, J. Ahn, and I. Kim, "Mobile hotspot network system for high-speed railway communications using millimeter waves," ETRI Journal, vol. 38, no. 6, pp. 1052-1063, 2016.

[2] C.-K. Jao, C.-Y. Wang, T.-Y. Yeh et al., "WiSE: a system-level simulator for $5 \mathrm{G}$ mobile networks," IEEE Wireless Communications, vol. 25, no. 2, pp. 4-7, 2018.

[3] R. Bifulco, A. Matsiuk, and A. Silvestro, "CATENAE: a scalable service function chaining system for legacy mobile networks," International Journal of Network Management, vol. 27, no. 2, pp. 1-14, 2017.

[4] J. Kim, M. Sung, S. H. Cho et al., "MIMO-supporting radioover-fiber system and its application in mmWave-based indoor 5G mobile network," Journal of Lightwave Technology, vol. 38, no. 1, pp. 101-111, 2020.

[5] F. Liu, Y. Liu, Y. Liu, and H. Wang, "A novel construction paradigm of multimedia awareness system for mobile network," Cluster Computing, vol. 22, Supplement 4, pp. 9697-9713, 2019. 
[6] J. Kim, M. Schmieder, M. Peter et al., "A comprehensive study on mmWave-based mobile hotspot network system for highspeed train communications," IEEE Transactions on Vehicular Technology, vol. 68, no. 3, pp. 2087-2101, 2019.

[7] H. Jarrah, N. I. Sarkar, and J. Gutierrez, "Comparison-based system-level fault diagnosis protocols for mobile ad-hoc networks: a survey," Journal of Network \& Computer Applications, vol. 60, no. 1, pp. 68-81, 2016.

[8] T. J. Gerpott and P. Meinert, "Correlates of using the billing system of a mobile network operator to pay for digital goods and services," Information Systems Frontiers, vol. 18, no. 6, pp. 1265-1283, 2016.

[9] B. Clerckx, A. Costanzo, A. Georgiadis, and N. B. Carvalho, "Toward 1G mobile power networks: RF, signal, and system designs to make smart objects autonomous," IEEE Microwave Magazine, vol. 19, no. 6, pp. 69-82, 2017.

[10] F. A. Khan, M. Imran, H. Abbas, and M. H. Durad, "A detection and prevention system against collaborative attacks in mobile ad hoc networks," Future Generation Computer Systems, vol. 68, no. 3, pp. 416-427, 2017.

[11] H. Al Moussawi, F. Fardoun, and H. Louahlia-Gualous, "Review of tri-generation technologies: design evaluation, optimization, decision-making, and selection approach," Energy Conversion and Management, vol. 120, no. 7, pp. 157-196, 2016.

[12] Z. Liang, J. Zang, X. Yang, X. Dong, and H. Song, "Integration interval determination and decision threshold optimization for improved TRPC-UWB communication systems," China Communications, vol. 14, no. 5, pp. 185-192, 2017.

[13] Q. Zhang, R. Wang, J. Yang, K. Ding, Y. Li, and J. Hu, "Collective decision optimization algorithm: a new heuristic optimization method," Neurocomputing, vol. 221, no. 1, pp. 123137, 2016.

[14] S. Sekizaki, T. Yamasaki, I. Nishizaki, T. Hayashida, H. Ishikawa, and H. Uenishi, "A development of evolutionary many-objective optimization method for decision aid on distribution system reconfiguration," IEEJ Transactions on Power and Energy, vol. 138, no. 12, pp. 925-938, 2018.

[15] D. Plets, K. Chemmangat, D. Deschrijver et al., "Surrogate modeling based cognitive decision engine for optimization of WLAN performance," Wireless Networks, vol. 23, no. 8, pp. 2347-2359, 2017.

[16] X. Gu, S. Li, H. Liang, and K. Li, "Optimization decisionmaking method for looped network restoration to eliminate transmission line overload in network reconfiguration," Proceedings of the CSEE, vol. 37, no. 5, pp. 1379-1388, 2017.

[17] M. Pouraminian and S. Pourbakhshian, "Multi-criteria shape optimization of open-spandrel concrete arch bridges: Pareto front development and decision-making," Military Operations Research, vol. 16, no. 5, pp. 670-680, 2019.

[18] S.-B. Tsai, "Using the DEMATEL model to explore the job satisfaction of research and development professionals in China's photovoltaic cell industry," Renewable and Sustainable Energy Reviews, vol. 2018, no. 81, pp. 62-68, 2018.

[19] H. Wang, Y. Huang, A. Khajepour, D. Cao, and C. Lv, "Ethical decision-making platform in autonomous vehicles with lexicographic optimization based model predictive controller," IEEE Transactions on Vehicular Technology, vol. 69, no. 8, pp. 81648175, 2020.

[20] Z. Nedělková, P. Lindroth, M. Patriksson, and A.B. Strömberg, "Efficient solution of many instances of a simulation-based optimization problem utilizing a partition of the decision space," Annals of Operations Research, vol. 265, no. 1, pp. 93-118, 2018.

[21] S.-B. Tsai, Y.-C. Lee, C.-H. Wu, and J.-J. Guo, "Examining how manufacturing corporations win orders," South African Journal of Industrial Engineering, vol. 24, no. 3, pp. 112-124, 2013.

[22] J. Li, S. Fong, S. Mohammed, J. Fiaidhi, Q. Chen, and Z. Tan, "Solving the under-fitting problem for decision tree algorithms by incremental swarm optimization in rare-event healthcare classification," Journal of Medical Imaging and Health Informatics, vol. 6, no. 4, pp. 1102-1110, 2016.

[23] W. Wu, Y. Liu, C. H. Wu, and S. B. Tsai, “An empirical study on government direct environmental regulation and heterogeneous innovation investment," Journal of Cleaner Production, vol. 254, article 120079, 2020.

[24] C. B. Xiao, D. Z. Feng, and M. D. Yuan, "Soft decision optimization method for robust fundamental matrix estimation," Machine Vision and Applications, vol. 30, no. 4, pp. 657-669, 2019.

[25] K. Meng, P. Lou, X. Peng, and V. Prybutok, "Multi-objective optimization decision-making of quality dependent product recovery for sustainability," International Journal of Production Economics, vol. 188, no. 6, pp. 72-85, 2017.

[26] M. Baldan, A. Nikanorov, and B. Nacke, "A novel multisurrogate multi-objective decision-making optimization algorithm in induction heating," COMPEL, vol. 39, no. 1, pp. 144-157, 2020.

[27] X. Li, J. Wen, and A. Malkawi, "An operation optimization and decision framework for a building cluster with distributed energy systems," Applied Energy, vol. 178, no. 9, pp. 98-109, 2016.

[28] A. Jafari and V. Valentin, "Selection of optimization objectives for decision-making in building energy retrofits," Building and Environment, vol. 130, no. 2, pp. 94-103, 2018.

[29] K. S. Bhattacharjee, H. K. Singh, M. Ryan, and T. Ray, "Bridging the gap: many-objective optimization and informed decision-making," IEEE Transactions on Evolutionary Computation, vol. 21, no. 5, pp. 813-820, 2017. 